\title{
Intelligent Transformer (I-Transformer)
}

\author{
Kiran Sara Thomas, Melbin Benny, Roy J Mathew, Sebin Jose
}

\begin{abstract}
Transformers plays a crucial role in the distribution and transmission system. In persisting electrical network, it is hard to manually monitor various conditions of each transformer. So, the requirement of automatic transformer condition monitoring and data acquisition has become a necessity. For the strong run of each nation reliable and safe supply of electricity is essential so the idea of an Intelligent Transformer is put forward. The proposed system comprises of design and implementation of a mobile embedded system to observe load current, voltage, oil level, temperature and humidity. The online monitoring system is executed by integrating the Internet of Things (IoT) with relays and sensors. The data from each sensor is processed and stored in system memory. The system checks for abnormal values in data received depending on predefined conditions and on occurrence it alerts the respected officials through front end application.

The system also consists of a fuzzy controller aided by certain gases formed from the transformer oil. By performing Dissolved Gas Analysis (DGA) on gases fault prediction and diagnostics can be performed on the transformer. The system focuses on improving the efficiency of the transformer monitoring process and provide a reliable supply network.
\end{abstract}

Index Terms-Distribution Transformer Monitoring, IoT, Fuzzy Controller, Dissolved Gas Analysis.

\section{INTRODUCTION}

Distribution Transformers is an electrical isolation transformer used to convert high-level voltage to low-level voltage necessary for domestic and commercial applications. So, the proper operation of the distribution transformer is an important criterion of an electrical distribution network. Under proper conditions, the transformer operates satisfactorily. However, the transformer life is impacted by heating, overloading, a various fault occurring in the electrical network. Improper functioning of the transformer leads to an unreliable and unsafe supply network. So, the requirement of monitoring transformer conditions regularly to maintain the absolute operation of the network. The present monitoring systems existing have deficiencies and issues. They measure single parameters such as power, current, voltage etc. While some measure multi-parameter

the time required in data acquisition and testing speed isvery high. Another drawback is that data obtained is not sent to

Kiran Sara Thomas, Department of Electrical and Electronics Engineering, APJ Abdul Kalam Technological University, Rajagiri School of Engineering and Technology, Ernakulum, India

Melbin Benny, Department of Electrical and Electronics Engineering, APJ Abdul Kalam Technological University, Rajagiri School of Engineering and Technology, Ernakulum, India

Roy J Mathew, Department of Electrical and Electronics Engineering, APJ Abdul Kalam Technological University, Rajagiri School of Engineering and Technology, Ernakulum, India

Sebin Jose, Department of Electrical and Electronics Engineering, APJ Abdul Kalam Technological University, Rajagiri School of Engineering and Technology, Ernakulum, India monitoring centres in time thus they couldn't judge the three-phase equilibrium of the transformer. Many monitoring systems use power communications to send the acquired data. They introduce difficulties such as electrical noise, frequency interference. So, we need a system that monitors all system parameters and sends data to monitoring centres in time. The proposed system provides online transformer health monitoring, data acquisition and fault prediction system. The system presents the realization of a cloud embedded system to monitor current, voltage, temperature, humidity and oil level. The system integrates the Internet of Things (IoT) with appropriate sensors and relays. The data obtained are processed and recorded in system memory. Based on predefined conditions the system check for abnormal conditions and on the occurrence of the proper actions are carried out. The fuzzy logic controller is implemented for incipient fault prediction and diagnosis fault prediction.

\section{LITERATURE SURVEY}

DeekshaSrivastava, AwanishKesarwani, ShivaniDubey, "put forward a system for measurement of Temperature and Humidity by using Arduino Tool and DHT sensor. The proposed system can provide a convenient method for effective monitoring of temperature and humidity in real-time.’[1]

V. Shinge, O.Shukla, P. Panday, and M.chaple, "proposed wireless transformer monitoring parameters. The most important purpose of this system is to monitor and control the transformer through RF modules. By using a temperature sensor, microcontroller and RF transmission which is wireless communication, both monitoring and controlling can be done. The transformer has three parameters that are monitored i.e. voltage, current and temperature. The monitored data is then sent to a remote location. The transmission between RF and client is done via Controller."[2]

Mohammad Yunus, SharminRahman, ArifulAlam, KhondokarNazmusSakib, proposed an Arduino Based Smart AC-DC Voltmeter for Electronic Circuit in which Arduino senses the input voltage, converts the analogue voltage into a digital value.[3]

Daniel Dorcea, MihaelaHnatiuc, Iulian Lazar, presents a method of calibrating gas sensors using LabView.The identification of the calibration equation is used to calibrate gas sensors. The methods used to identify the calibration are linear regression and samples compare.[4]

Nicodemus M. Sakayo, Joseph N. Mutuku, James M. Ngaruiya, developed a microcontroller-based MQ-4 gas sensor for domestic cooking gas systems. The MQ-4 sensor has been used to detect the leakage of cooking gas which is mostly methane gas. The simplified electrical circuit of MQ 
gas sensors and sensing parameters are presented in this paper.[5]

DeepikaBhalla, Raj Kumar Bansal, and Hari Om Gupta, provide the design and implementation of the Dissolved Gas Analysis (DGA) method to identify faults by considering the ratios of specific dissolved gas concentrations in the transformer mineral oil. The data is analyzed using the IEC ratio method.[6]

Nitin K Dhote and Jagdish B Helonde, provide us with the implementation of the fuzzy controller for Dissolved Gas Analysis (DGA) using the IEC method. Also provided with the test cases for accurate measurement.[7]

\section{SYSTEM ARCHITECTURE}

In this fast-developing world, the need for a reliable and safe electrical supply network is augmenting and our projected system ensures a smooth, secure and swift supply of power to consumers. Various parameters are subjected to measure and promoting online measuring than manual measurement make the system superior. A real-time IoT based system is developed for this purpose and in addition to it, Fault prediction analysis and diagnosis is performed in the distribution transformer.

\section{A. The Objective of the Problem}

1) The projected system prevents the occurrence of faults and losses in the power supply which ominously benefits utility consumers.

2) Provides Self-protection in case of fault on the distribution transformer and the separation of the transformer and inform the operations teams.

3) Employed to monitor multiple transformers closely.

4) Enhance system stability and reliability by the monitoring system.

5) Avoid the occurrence of power and data losses as wireless communication is achieved.

6) The usage of Wi-Fi aids to achieve more accuracy and quick response in fault monitoring.

7) Fault prediction and diagnosis of transformer performing Dissolved Gas Analysis.

\section{B. Proposed System Architecture}

Various parameters such as current, voltage, temperature, humidity and gas level are measured using the various sensors and a microcontroller is employed in the system to perform computation. Real-time monitoring is achieved in the system using the IoT platform provided and the Wi-Fi module is worked to achieve wireless communication. Operation officials in control rooms are updated about the transformer condition regularly and during the occurrence of fault depending on predefined instructions relay employed in the system gets activated and protect the transformer. Elimination of manual examination of transformer and labour cost could be attained. The gas sensors employed in system support to perform Dissolved Gas Analysis (DGA) and predict the occurrence of Incipient fault using the Fuzzy Controller designed based on the various gas concentration.

\section{Basic Block Diagram of System}

The block diagram shown below represents the monitoring device system proposed mounted near the LV side of the distribution transformer.

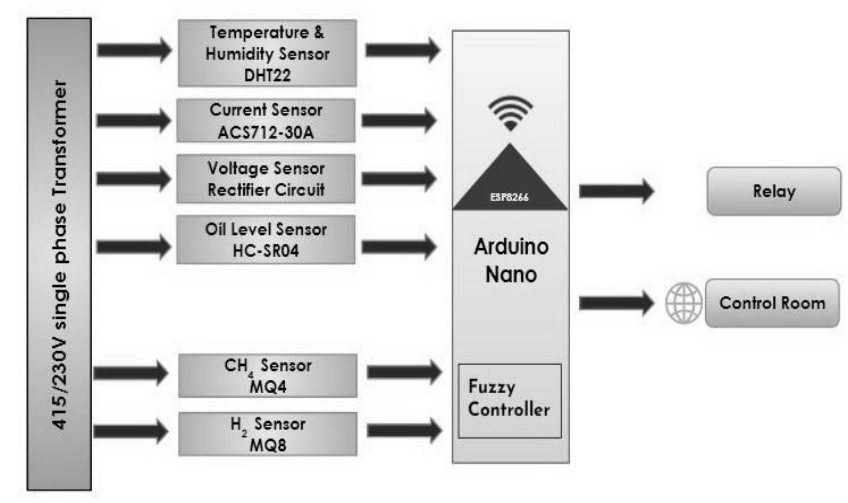

Fig.1. Block Diagram of the system

The below figure shows the flow chart of the proposed system.

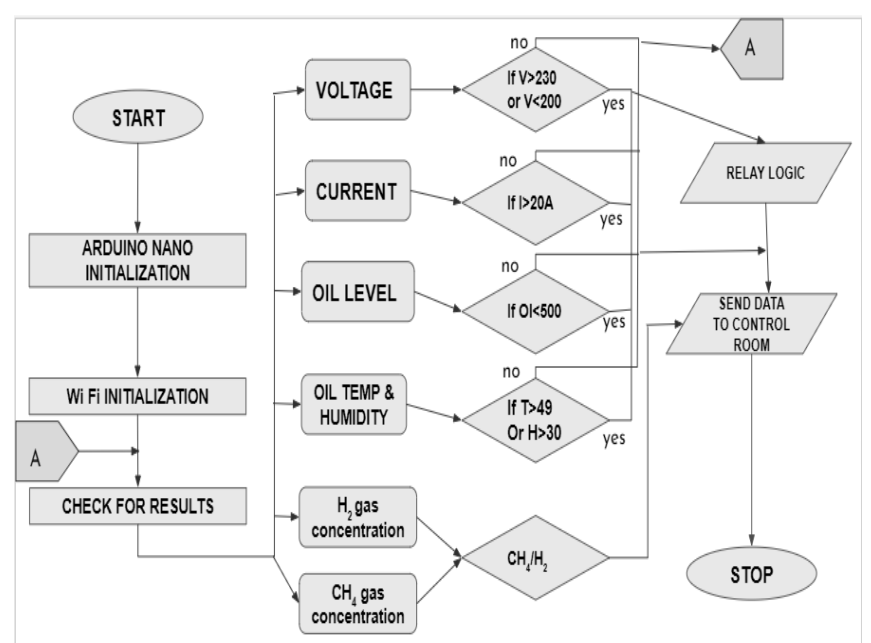

Fig.2. flow chart of the proposed system

The below figure shows the simulation diagram of the proposed system developed using Proteus software. Here wire labels are provided to avoid messy connections.

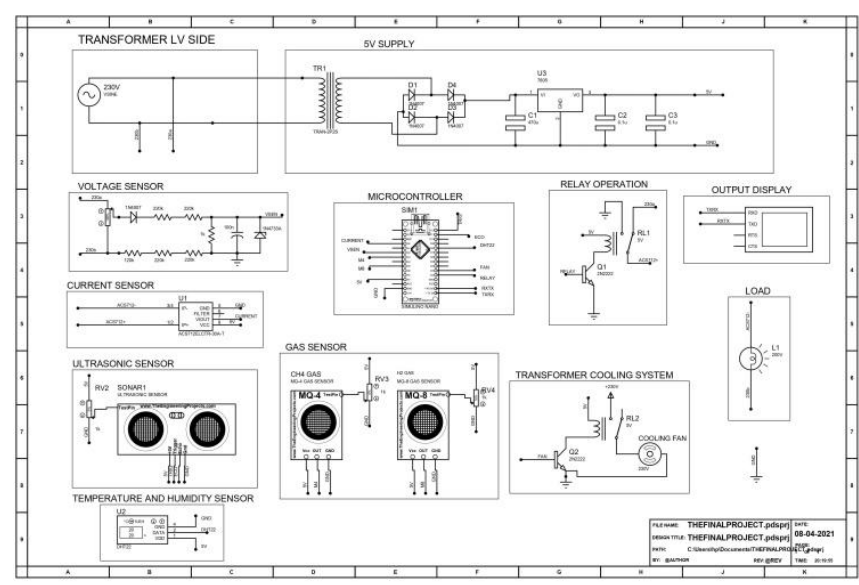

Fig.3. simulation diagram of the proposed system 


\section{HARDWARE IMPLEMENTATION}

\section{A. 5V Supply Circuit}

We use $230 \mathrm{~V}, 50 \mathrm{~Hz}$ AC supply in general but this voltage has to be altered for the devices to operate. The microcontroller provided operates on a 5V DC supply so the AC supply should be converted to a $5 \mathrm{~V}$ supply using the step-down converter provided in the power supply circuit. The supply circuit is provided at the LV side of the transformer. The voltage at the LV side is stepped down using a step-down transformer to the required level. The AC power is then converted to DC using a Power Electronic Rectifier circuit. The circuit consists of a Bridge rectifier circuit for power conversion. The pulsating DC output is with ripples is filtered using a Capacitor Filter. To obtain the required $5 \mathrm{~V}$ step-down converter called Voltage Regulator IC7805 is used. The 5V supply circuit has been shown in the below figure.

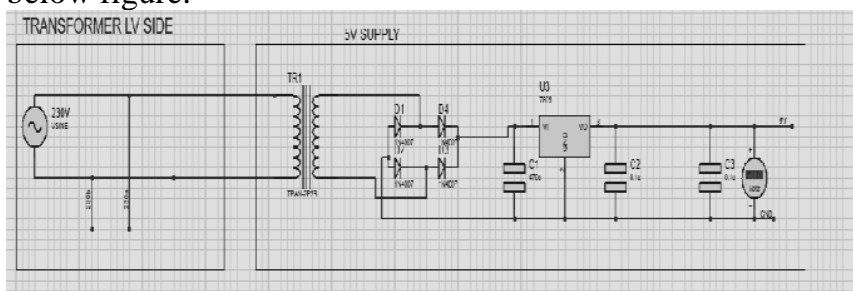

Fig.4. 5V Supply-Circuit

\section{B. Voltage Sensor}

The voltage sensor is used to measure and monitor the voltage value in the transformer. The sensor provided at the LV side of the transformer measures voltage value regularly and checks whether the voltage value exceeds the critical value. The sensor comprises of rectification element, a Potential Divider circuit and a Regulation device and the output of the sensor is fed to the microcontroller which reads the corresponding value and displays the original value.

The below figure shows the circuit diagram for the voltage sensor.

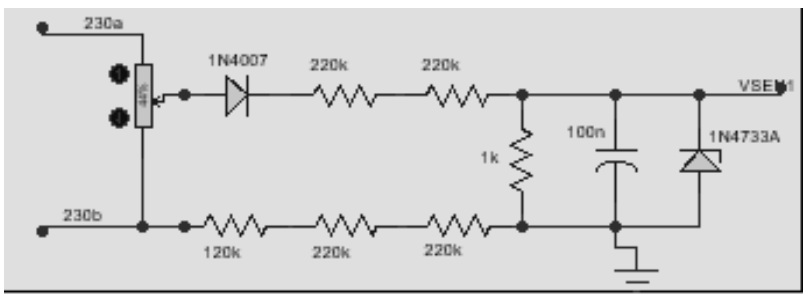

Fig.5. Voltage Sensor

\section{Current Sensor}

In the proposed system, the current is measured by using sensor ACS712-30A. The sensor yields accurate current measurements. Thus power consumption of the Overall system is monitored. The sensor provides an output voltage proportional to the observed current, based on the Hall Effect principle. In the ACS712 sensor, 5V supply is connected to $\mathrm{Vcc}$ and the ground of the sensor is connected to the ground of the Arduino. ACS712 module can measure current AC or $\mathrm{DC}$ ranging from $+30 \mathrm{~A}$ to $-30 \mathrm{~A}$. Vout generates an output that reflects the current flowing through the sensing pads until it is turned up. If the load is turned off, a ( Vcc/2) will be produced. The ACS712 will calculate current in both directions, with a voltage greater than $2.5 \mathrm{~V}(\mathrm{Vcc} / 2)$ indicating one direction and a voltage less than $2.5 \mathrm{~V}(\mathrm{Vcc} / 2)$ indicating the other. The analogue voltage given out by the ACS712 module is read using an analogue pin on the Arduino. The below figure shows the ACS712-30A current sensor.

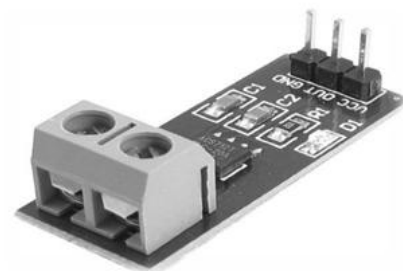

Fig.6. ACS712-30A Current sensor

\section{Ultrasonic Sensor}

In this proposed system, the sensor used is the HC-SR04 an ultrasonic sensor is shown in the below figure. The Ultrasonic transmitter transmits an ultrasonic wave, this wave travels in the air and when it gets objected by any material it gets reflected toward the sensor this reflected wave is observed by the Ultrasonic receiver module. The transmitter will emit a $40 \mathrm{kHz}$ ultrasonic pulse, and the receiver will wait for it to return. As the wave returns to the sensor after being mirrored by another source, the Echo pin goes high for a period of time relative to the time it took the wave to return to the sensor.

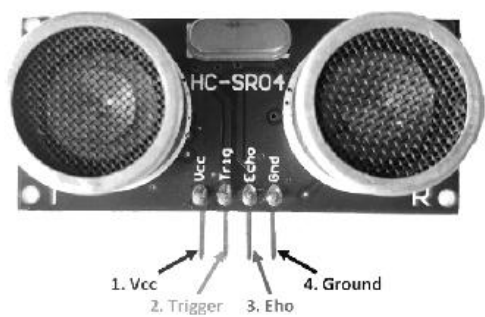

Fig.7. Ultrasonic Sensor

\section{E. Temperature and Humidity Sensor}

In this project, the DHT22 is used to measure the temperature, humidity as shown in the below figure. The DHT22 temperature range is from -40 to 80 degrees Celsius with \pm 0.5 degrees accuracy. The humidity range is from 0 to $100 \%$ with $5 \%$ accuracy. For the sampling rate, the DHT22 is $0.5 \mathrm{~Hz}$ or one reading every two seconds, and the DHT22 has a smaller body size. The operating voltage of both sensors is from 3 to 5 volts, while the max current used when measuring is $2.5 \mathrm{~mA}$. DHT22 sensor has four pins- VCC connected to $5 \mathrm{~V}$ of Arduino, GND connected to the ground of Arduino, Data Pin to a digital pin and Not connected pin is not used.

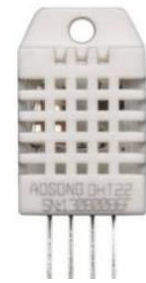

Fig.8. DHT11 temperature \& Humidity Sensor

\section{F. Gas Sensors}

Gas sensors are utilized to detect the concentration of various gases in the transformer tank. Depending on the various gas concentration ratio, a Fuzzy controller is created. MQ sensors are made used for this purpose. MQ sensors are metal oxide semiconductor which detects gas presence when the resistance of the sensing material varies as it comes in 
contact with the gas. MQ4 and MQ8 sensors are proposed in the system. MQ4 sensor has sensitivity towards methane, propane and butane whereas MQ8 has sensitivity towards Hydrogen. Below the figure shown is the MQ sensor.

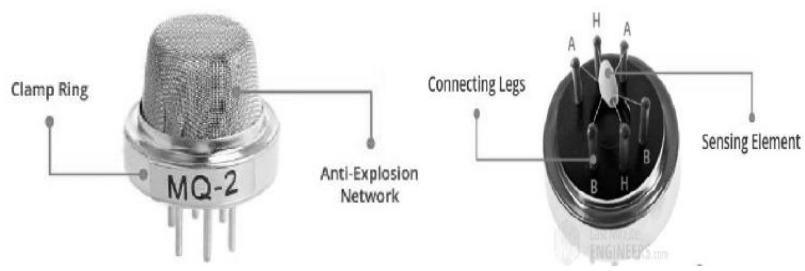

Fig.9. MQ Sensor

\section{G. Arduino Nano}

In this project, we use ATmega328 as the microcontroller incorporated in the Arduino Nano. It has a clock speed of $16-\mathrm{MHz}$ and an operating voltage of $5 \mathrm{~V}$. It consists of 8 analogue pins and 22 digital pins. The power consumption of the module is $19 \mathrm{~mA}$. It comes up with characteristics such as highly flexible, easy to use and cheap.

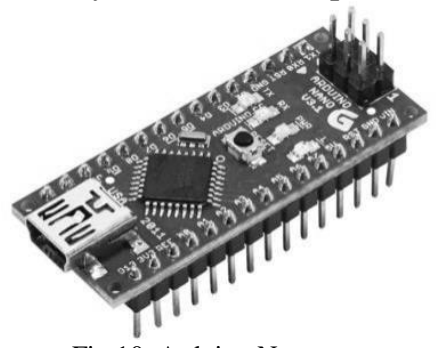

Fig.10. Arduino Nano

\section{H. Relay module}

In this project, the relay module used is SRD-05VDC-SL-C as shown below figure. When current flows through the coil of the relay, a magnetic field is created that causes a ferrous armature to move, either making or breaking an electrical connection.

When the electromagnet is energized the "NO" contact is the one that is on and the "NC" contact is the one that is off. Bipolar Transistor 2N2222 acts as a switch that is used to control the relay. The Relay is used to open and close the connection from the transformer to load according to the instructions from the microcontroller. The relay connections are provided as shown below figure.

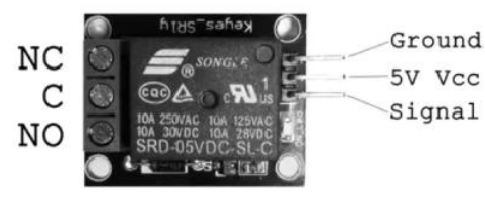

Fig.11. Relay Module

\section{Wi-Fi Module}

Wi-Fi module is employed for communication purpose. Its function is to convert serial port into an embedded module which can be conforming to wireless network communication standard as Wi-Fi. We utilize the ESP8266 Wi-Fi module shown below figure, as it offers a self-containing and complete Wi-Fi networking solution. It can either host the application or offloading all Wi-Fi networking functions from another application processor. Connectivity to the microprocessor is achieved through UART Interface. Performance and Memory requirement is improvised through cache. Another advantage of this module is that it is designed to occupy a minimal PCB area.

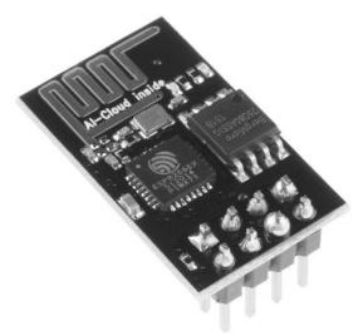

Fig.12. ESP8266 Wi-Fi Module

\section{SOFTWARE REQUIREMENTS}

\section{A. Arduino IDE}

The open-source Arduino software makes it convenient to write code and upload it to the board. This software can be used with any Arduino board. The fuzzy rule-based codes were also incorporated using this software. The below figure shows the ArduinoIDE software.

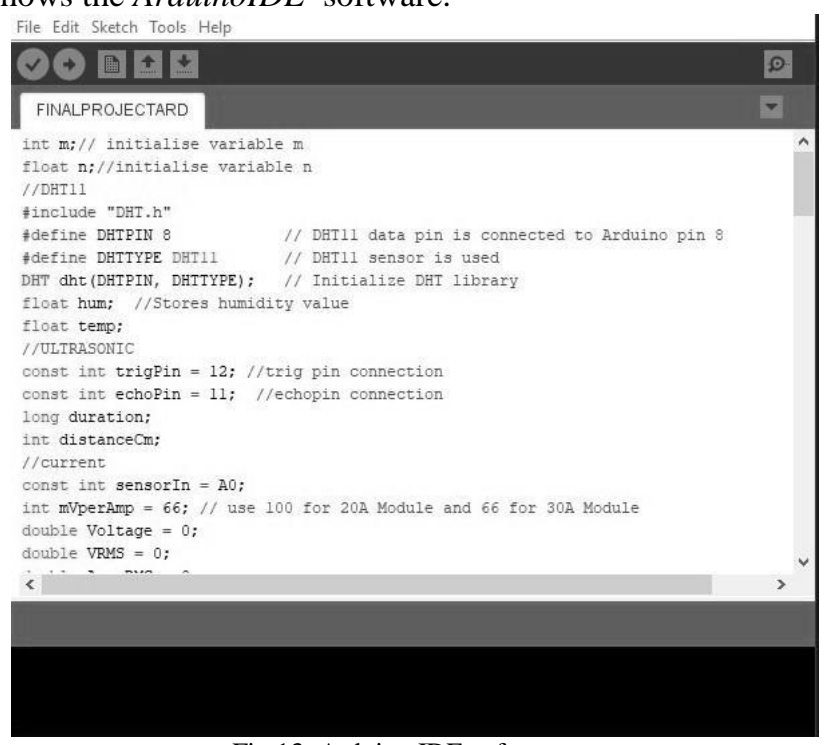

Fig.13. Arduino IDE software

\section{B. Internet of things (IoT)}

The Internet of Things (IoT) is a network of interconnected computing systems, mechanical and digital computers, and objects that can send and receive data without involving human-to-human or human-to-computer interaction. Here the software used is ThingSpeak.com as shown in the below figure.ThingSpeak is an IoT Cloud platform where you can send sensor data to the cloud operated by MathWorks.

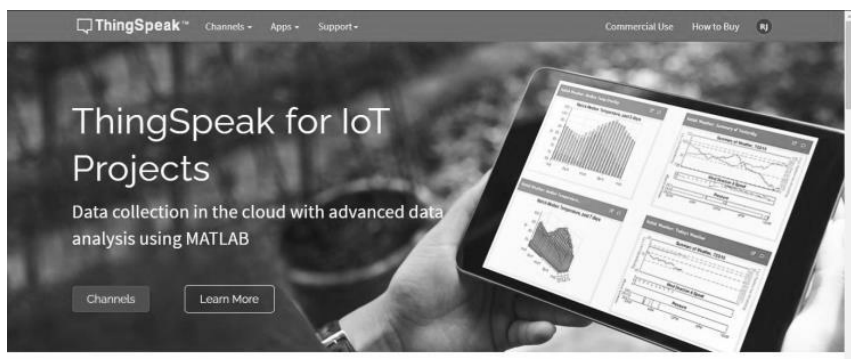

Fig.14.Thingspeak.com IoT Platform

\section{MATLAB}

MATLAB is a high-performance language for technical computing. It integrates computation, visualization, and programming in an easy-to-use environment where problems and solutions are expressed in familiar mathematical 
notation. Here the MATLAB is used to design the fuzzy controller for the fault diagnostic of the transformer.

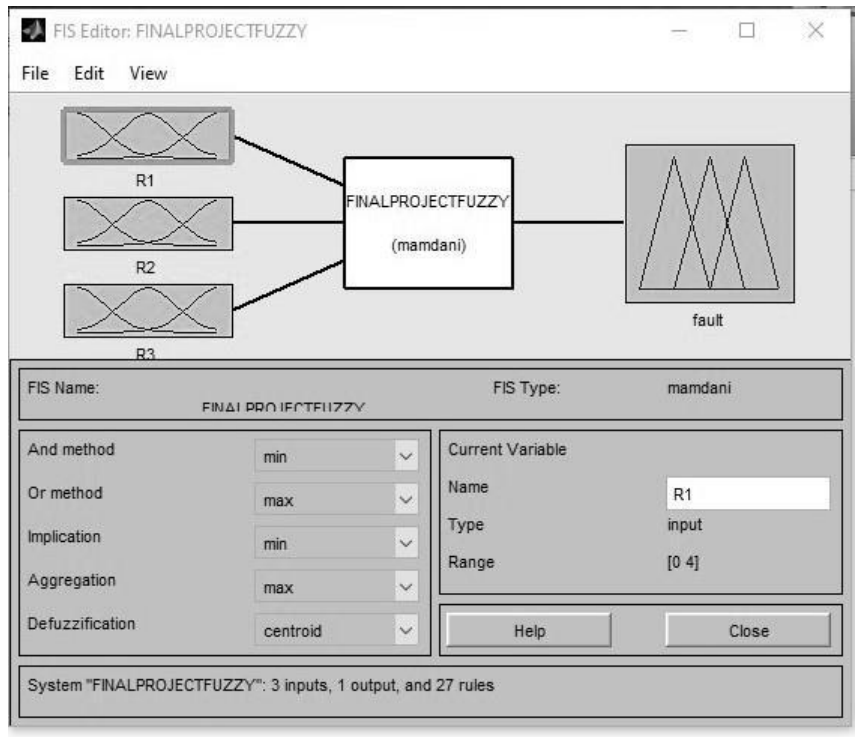

Fig.15.fuzzy controller designing using MATLAB.

\section{WORKING PRINCIPLE}

All the sensors and relay integrated into the LV side of the distribution transformer unit are interfaced with an Arduino microcontroller to receive and process various transformer parameters such as voltage, current, temperature, humidity, oil level and gas ratios are obtained. The project is divided into two parts as transformer condition monitoring and the other part as the fault predicting and diagnostic system, the above-mentioned parameters are processed and monitored and are been wirelessly transferred using wifi module to the IoT platform as mentioned before. here the DGA is done using the IES method. In the IES method, we consider 3 gas ratios and provides a fault weight, for the ease of analysis in this proposed project a single gas ratio has been considered ie. $\mathrm{R}_{2}$ is the ratio of $\mathrm{CH}_{4}$ and $\mathrm{H}_{2}$. The below tables shows the categorization of gas ratios and fault level [7]. From these fault weights, we can predict the future incipient fault can be predicted and the system will suggest the maintenance action. The values obtained by different sensors are sent to the IoT Cloud platform called ThingSpeak.com. With the help of a cloud platform, the data can be stored easily and it can be retrieved by authorities in the control room to monitor the transformer health conditions.

\begin{tabular}{|l|l|}
\hline Gas Ratio Code & Gas ratio \\
\hline $\mathrm{R}_{1}$ & $\mathrm{C}_{2} \mathrm{H}_{4} / \mathrm{C}_{2} \mathrm{H}_{6}$ \\
\hline $\mathrm{R}_{2}$ & $\mathrm{CH}_{4} / \mathrm{H}_{4}$ \\
\hline $\mathrm{R}_{3}$ & $\mathrm{C}_{2} \mathrm{H}_{2} / \mathrm{C}_{2} \mathrm{H}_{4}$ \\
\hline
\end{tabular}

Table.1. Gas ratios

\begin{tabular}{|l|l|l|}
\hline Fault Type & $\begin{array}{l}\text { Characteristic } \\
\text { Fault }\end{array}$ & Fault weights \\
\hline TL & $\begin{array}{l}\text { Thermal Fault of } \\
\text { low temperature }\end{array}$ & $<0.2>$ \\
\hline PD & $\begin{array}{l}\text { Partial Discharge } \\
\text { of high energy } \\
\text { density }\end{array}$ & $<0.4>$ \\
\hline D1 & $\begin{array}{l}\text { Discharge of low } \\
\text { energy }\end{array}$ & $<0.6>$ \\
\hline
\end{tabular}

\begin{tabular}{|l|l|l|}
\hline TH & $\begin{array}{l}\text { Thermal Fault of } \\
\text { high temperature }\end{array}$ & $\langle 0.8\rangle$ \\
\hline D2 & $\begin{array}{l}\text { Discharge of high } \\
\text { energy }\end{array}$ & $<1.0\rangle$ \\
\hline
\end{tabular}

Table.2. Types of Faults and their Weights

\section{EXPERIMENTAL RESULTS}

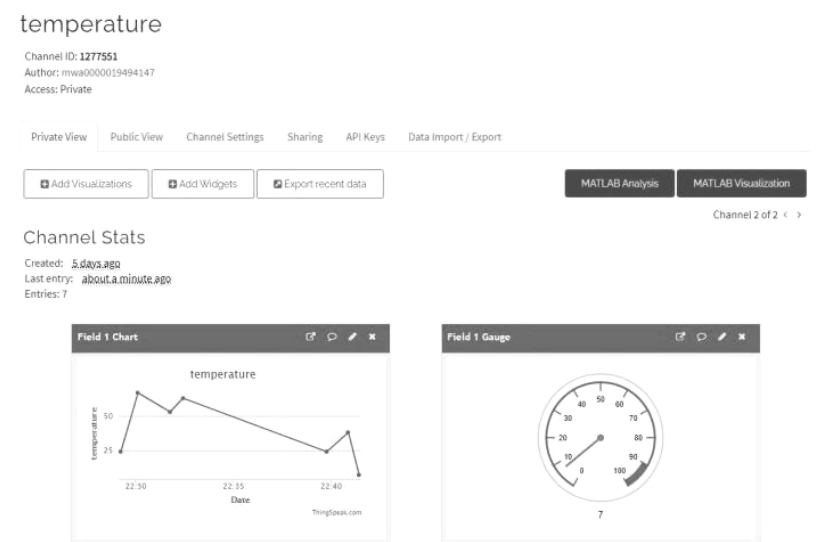

Fig.16. Temperature Output of Transformer

The above figure shows the result of the temperature output of the transformer in the IoT platform. The below figure shows the test result of the gas ratio of $\mathrm{CH}_{4}$ and $\mathrm{H}_{2}$.

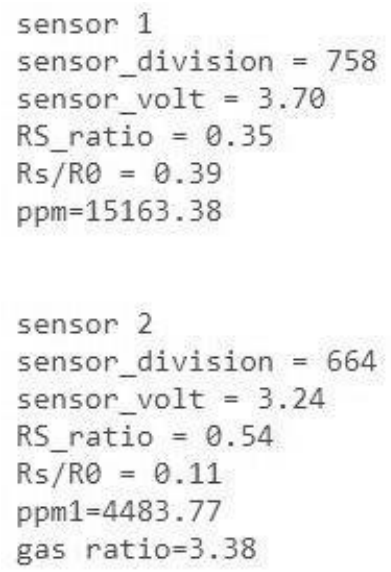

Fig.17. result of gas ratio

Here shows the working of the fuzzy controller diagnosing the fault and suggesting a maintenance plan.

Concentrations and ratios of dissolved gases in ppm are found as follows:

$\mathrm{C}_{2} \mathrm{H}_{2}-26 ; \mathrm{C}_{2} \mathrm{H}_{4}-23 ; \mathrm{CH}_{4}-2221 ; \mathrm{H}_{2}-2257 ; \mathrm{C}_{2} \mathrm{H}_{6}-22$

$$
R_{1}-1.13 ; R_{2}-0.0979 ; R_{3}-1.05
$$

and the fault weight is 0.5 which is D1.[7] The below figure shows the result based on the rules provided for the fuzzy controller. 
$\mathrm{R} 1-1.13$

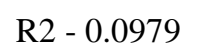

$\mathrm{R} 3-1.05$

fault -0.5
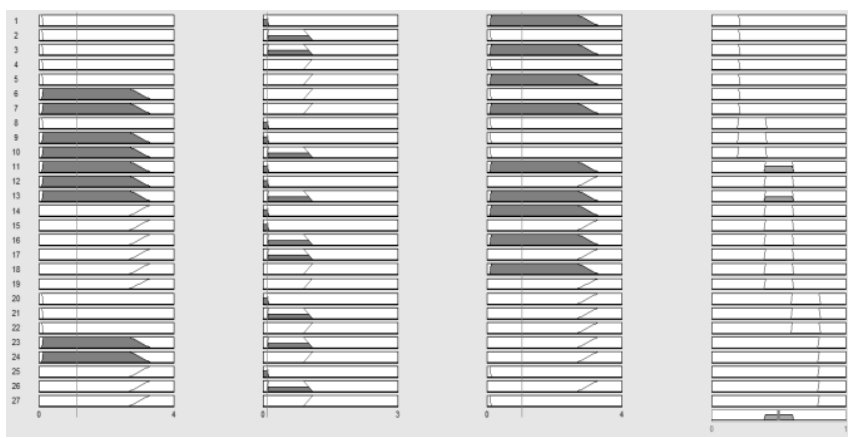

Fig.18.MATLAB output of the fault weight

The defects are of medium intensity. Monthly oil testing and determining load dependency are recommended maintenance actions.[7]

\section{ACKNOWLEDGMENT}

We are highly indebted to Dr. Elizabeth Rita Samuel, Head of the department Electrical and Electronics Engineering, for guiding us throughout the process. A special thanks to Mr.Jebin Francis, Asst. Professor, Department of Electrical Engineering, for piloting the main project through trials and tribulations. Our heartfelt thanks to our project guide $\mathrm{Mr}$. Thomas K.P for spending her precious time with us.We also remember our teachers of the Department of Electrical Engineering, who were always supportive of our academics. We are also thankful to our friends and well-wishers for their support and prayers. With a heart full of gratitude we submit this paper.

\section{CONCLUSION}

Transmission of energy from producing power stations to end-users is the primary aim of power distribution. Distribution system networks transmit energy and deliver it to its load centres. Thus, a distribution network must possess high efficiency, reliability and service quality.

The project focuses on enhancing the performance of the distribution transformer monitoring process. The whole system ensures high transmission reliability and performance. The suggested scheme is cost-effective and reduces the likelihood of human error in transformer control scenarios. Relays embedded with different sensors provide adequate reliability and security with improved performance and the Wi-Fi module assist in sending the operation details with the aid of the IoT platform to the control room where the required data is stored in the cloud platform. The data received will help the utility to keep a check on the operational behaviour of the transformers and identify faults before any failures. In addition, the system provides a pathway to tackle with necessary measures in case of emergency to the transformers. Also, data Incipient Fault prediction and diagnosis of transformer faults using Fuzzy logic are implemented.

\section{REFERENCES}

[1] DeekshaSrivastava, AwanishKesarwani and ShivaniDubey," Measurement of Temperature and Humidity by using Arduino Tool and DHT11", International Research Journal of Engineering and Technology (IRJET), 2008, pp.876-878.
[2]V. Shinge , O.Shukla , P. Panday, and M.chaple, "Wireless Transformer Parameter Monitoring System Using RF Module".International Journal of Innovative Research in Electrical, Electronics, Instrumentation and Control Engineering Vol. 4, Issue 4, April 2016.

[3]Mohammad Yunus, SharminRahman, ArifulAlam and KhondokarNazmusSakib," Arduino Based Smart AC-DC Voltmeter for Electronic Circuit Analysis", IOSR Journal of Electrical and Electronics Engineering (IOSR-JEEE) e-ISSN: 2278-1676,p-ISSN: 2320-3331, Volume 15, Issue 1 Ser. I (Jan-Feb 2020), PP 10-15

[4]Daniel Dorcea, MihaelaHnatiuc, Iulian Lazar Electronic and Telecommunication Department Constanta Maritime University Constanta, Romania."Acquisition and Calibration Interface for Gas Sensors" in proceedings of 2018 IEEE 24th International Symposium for Design and Technology in Electronic Packaging (SIITME).

[5]Nicodemus M. Sakayo, Joseph N. Mutuku, James M. Ngaruiya, Department of Physics, Jomo Kenyatta University of Agriculture and Technology." Design and Calibration of a Microcontroller Based MQ-4 Gas Sensor for Domestic Cooking Gas System" in SSRG International Journal of Applied Physics (SSRG-IJAP) - Volume 6 Issue 2- May to August 2019.

[6] DeepikaBhalla, Raj Kumar Bansal, and Hari Om Gupta "Transformer Incipient Fault Diagnosis Based on DGA using Fuzzy Logic" Indian International Conference on Power Electronics 2010 (IICPE-2010). 10 March 2011.

[7] Nitin K Dhote and Jagdish B Helonde "Fuzzy system for transformer fault diagnosis and maintenance using DGA" Journal of Electrical Engineering(J ELECTR ENG-SLOVAK) January 2014. 